\title{
Inflammation and acute traffic-related air pollution exposures among a cohort of youth with type 1 diabetes ${ }^{\text {is }}$
}

\author{
Robin C. Puett ${ }^{\mathrm{a}, *}$, Jeff D. Yanosky ${ }^{\mathrm{b}}$, Murray A. Mittleman ${ }^{\mathrm{c}}$, Jessica Montresor-Lopez ${ }^{\mathrm{a}}$, \\ Ronny A. Bell ${ }^{\mathrm{d}}$, Tessa L. Crume ${ }^{\mathrm{e}}$, Dana Dabelea ${ }^{\mathrm{e}}$, Lawrence M. Dolan ${ }^{\mathrm{f}}$, Ralph B. D'Agostino Jr ${ }^{g}$, \\ Santica M. Marcovina ${ }^{\text {h }}$, Catherine Pihoker ${ }^{i}$, Kristi Reynolds $^{j}$, Elaine Urbina ${ }^{k}$, Angela D. Liese ${ }^{1}$ \\ ${ }^{a}$ Maryland Institute for Applied Environmental Health, University of Maryland School of Public Health, College Park, MD, USA \\ ${ }^{\mathrm{b}}$ Division of Epidemiology, Department of Public Health Sciences, Pennsylvania State University College of Medicine, Hershey, PA, USA \\ ${ }^{\mathrm{c}}$ Department of Epidemiology, TH Chan Harvard School of Public Health, Boston, MA, USA \\ ${ }^{\mathrm{d}}$ Department of Public Health, Brody School of Medicine, East Carolina University, Greenville, NC 27834, USA \\ ${ }^{\mathrm{e}}$ Department of Epidemiology, Colorado School of Public Health, University of Colorado-Denver Anschutz Medical Center, Denver, CO, USA \\ ${ }_{\mathrm{f}}^{\mathrm{f}}$ Division of Pediatric Endocrinology, Cincinnati Children's Hospital Medical Center, Cincinnati, OH, USA \\ ${ }^{\mathrm{g}}$ Department of Biostatistical Sciences, Wake Forest University School of Medicine, Winston-Salem, NC, United States \\ ${ }^{\mathrm{h}}$ Division of Metabolism, Endocrinology and Nutrition, Northwest Lipid Metabolism and Diabetes Research Laboratories, Seattle, WA, USA \\ ${ }^{\mathrm{i}}$ Department of Pediatrics, University of Washington, Seattle, USA \\ ${ }^{\mathrm{j}}$ Department of Research \& Evaluation, Kaiser Permanente Southern California, Pasadena, CA, USA \\ ${ }^{\mathrm{k}}$ Heart Institute, Cincinnati Children's Hospital Medical Center, Cincinnati, OH, USA \\ ${ }^{1}$ Department of Epidemiology and Biostatistics, Arnold School of Public Health, University of South Carolina, Columbia, SC, USA
}

\section{A R T I C L E I N F O}

Handling Editor: Hanna Boogaard

Keywords:

Traffic-related air pollution

Inflammation

Diabetes

\begin{abstract}
A B S T R A C T
Background: Evidence remains equivocal regarding the association of inflammation, a precursor to cardiovascular disease, and acute exposures to ambient air pollution from traffic-related particulate matter. Though youth with type 1 diabetes are at higher risk for cardiovascular disease, the relationship of inflammation and ambient air pollution exposures in this population has received little attention.

Objectives: Using five geographically diverse US sites from the racially- and ethnically-diverse SEARCH for Diabetes in Youth Cohort, we examined the relationship of acute exposures to $\mathrm{PM}_{2.5}$ mass, Atmospheric Dispersion Modeling System (ADMS)-Roads traffic-related PM concentrations near roadways, and elemental carbon (EC) with biomarkers of inflammation including interleukin-6 (IL-6), c-reactive protein (hs-CRP) and fibrinogen.

Methods: Baseline questionnaires and blood were obtained at a study visit. Using a spatio-temporal modeling approach, pollutant exposures for 7 days prior to blood draw were assigned to residential addresses. Linear mixed models for each outcome and exposure were adjusted for demographic and lifestyle factors identified a priori.

Results: Among the 2566 participants with complete data, fully-adjusted models showed positive associations of EC average week exposures with IL-6 and hs-CRP, and $\mathrm{PM}_{2.5}$ mass exposures on lag day 3 with IL- 6 levels. Comparing the 25th and 75th percentiles of average week EC exposures resulted in 8.3\% higher IL-6 (95\%CI: $2.7 \%, 14.3 \%$ ) and $9.8 \%$ higher hs-CRP (95\%CI: $2.4 \%, 17.7 \%)$. We observed some evidence of effect modification for the relationships of $\mathrm{PM}_{2.5}$ mass exposures with hs-CRP by gender and with IL- 6 by race/ethnicity. Conclusions: Indicators of inflammation were associated with estimated traffic-related air pollutant exposures in this study population of youth with type 1 diabetes. Thus youth with type 1 diabetes may be at increased risk of air pollution-related inflammation. These findings and the racial/ethnic and gender differences observed deserve further exploration.
\end{abstract}

\footnotetext{
Competing financial interests: none.

* Corresponding author at: Public Health Bldg Rm 2234EE, Maryland Institute for Applied Environmental Health, University of Maryland School of Public Health, College Park, MD, USA.

E-mail address: rpuett@umd.edu (R.C. Puett).
} 


\section{Introduction}

There is persuasive evidence that exposures to ambient air pollution are associated with increased risk of cardiovascular disease morbidity and mortality (Dockery et al., 1993; Laden et al., 2006; Miller et al., 2007; Pope et al., 2002; Puett et al., 2008). Proposed biological mechanisms underlying this relationship include changes in autonomic function, oxidative stress, and systemic inflammation leading to endothelial dysfunction, thrombosis or atherosclerosis (Gurgueira et al., 2002; Pope et al., 2004; Pope and Dockery, 2006; Souza et al., 1998; Utell et al., 2002).

Individuals with diabetes bear a disproportionately greater risk of cardiovascular disease morbidity and mortality associated with increases in air pollution (Dubowsky et al., 2006; Kan et al., 2007; Maynard et al., 2007; O'Neill et al., 2005; Ostro et al., 2006; Peel et al., 2007). Studies have shown that cardiovascular hospitalizations and emergency department visits associated with ambient air pollution exposures are higher in persons with diabetes compared to persons without diabetes (Peel et al., 2007; Pereira Filho et al., 2008; Zanobetti and Schwartz, 2001, 2002). However, why individuals with diabetes are particularly vulnerable to the effects of ambient air pollution is poorly understood. Evidence suggests mechanisms associated with proinflammatory responses (Jacobs et al., 2010), chronic inflammation/oxidative stress, chronic autonomic dysfunction resulting in arterial stiffness, or imbalances in arterial vasoactive mediators (Gold, 2008). The vast majority of air pollution-related research in persons with diabetes has been conducted in adults - who largely have type 2 diabetes (T2D). Among this population, findings have been equivocal, particularly with respect to size fractions of particulate matter (PM) (e.g., one study found a relationship with $\mathrm{PM}_{0.25}$ but not with larger size fractions (Delfino et al., 2009)). Some studies observed an association of PM exposures with inflammatory biomarkers: interleukin-6 (IL-6), Creactive protein (CRP), or fibrinogen; while other studies have reported no relationship (Rich et al., 2012; Tsai et al., 2012; Williams et al., 2011; Zeka et al., 2006).

Few published studies have addressed the impact of air pollution on youth with diabetes, the vast majority of whom have type 1 diabetes (T1D). This research includes a few studies of air pollution exposures and diabetes incidence (Di Ciaula, 2016; Hathout et al., 2002, 2002; Malmqvist et al., 2015; Michalska et al., 2017) or metabolic control (Lanzinger et al., 2018; Tamayo et al., 2016), and a study of the relationship of air pollution exposures with oxidative stress, insulin resistance, and inflammation (Kelishadi et al., 2009). In a study conducted among Iranian youth aged 10-18 years, Kelishadi et al. (2009) reported that daily concentrations of $\mathrm{PM}_{10}$ averaged over the week prior to biomarker assessment were independently associated with increased high-sensitivity CRP (hs-CRP) ( $\beta: 1.5$ [SE: 0.2], $p<0.0001$ ) (Kelishadi et al., 2009).

Using data from the SEARCH for Diabetes in Youth Study (SEARCH), we examined whether inflammatory biomarkers (IL-6, hsCRP, and fibrinogen) were associated with acute exposure to trafficrelated air pollution including daily $\mathrm{PM}_{2.5}$ mass, Atmospheric Dispersion Modeling System (ADMS)-Roads (near roadway) estimated traffic-related $\mathrm{PM}$ concentrations, and $\mathrm{PM}_{2.5}$ elemental carbon (EC) among youth with T1D.

\section{Methods}

\subsection{Study population}

Participants were drawn from five of six original sites (Hawaii excluded) in the SEARCH for Diabetes in Youth Study (SEARCH), a large multicenter observational study of youth under aged 20 at the time of physician-diagnosed nongestational diabetes: South Carolina (statewide); eight counties surrounding Cincinnati, Ohio, including Butler, Clermont, Hamilton, Warren counties in Ohio, Boone, Campbell, and
Kenton counties in Kentucky, and Dearborn, Indiana); Colorado (statewide); Washington (5 counties surrounding Seattle, King, Kitsap, Pierce, Snohomish and Thurston) and health plan membership in 7 counties of Southern California (Los Angeles, Orange, Riverside, San Bernardino, Ventura, Imperial, Kern). Methods for the study are described in detail elsewhere (SEARCH Study Group, 2004; Hamman et al., 2014). Briefly, participants were identified via pediatric endocrinologists and health plan membership discharge diagnoses, laboratory measures and prescriptions. Data collection, including a baseline visit and follow-up visits at 12, 24 and 60 months, began for prevalent cases in 2001 and in 2002 for incident cases and is ongoing. Study visits were conducted under conditions of metabolic stability and in the absence of recent acute infection (Alman et al., 2014). For the current study, we used data from baseline visits from 2002 to 2006 for prevalent and incident cases. Case ascertainment is based on verification of a physician diagnosis of diabetes. Diabetes type was based on clinical type assigned by the health care provider as recorded in medical records. Information from baseline study visits included ascertainment of clinical and family history (parental education, income, etc.), information on treatment of diabetes, quality of life, health behaviors including diet and physical activity, anthropometric, blood pressure, and laboratory measures.

\subsection{Outcomes}

Plasma samples from blood drawn at the baseline study visit were used to analyze IL-6, hs-CRP, and fibrinogen. Participants were asked to fast for $8 \mathrm{~h}$ prior to the visit. Concentration of IL-6 in plasma was measured by a monoclonal antibody-based, high sensitive solid-phase ELISA method (R\&D System, Inc.). The assay sensitivity is $0.039 \mathrm{pg} / \mathrm{mL}$ and the interassay CVs of the low-, medium-, and high-level quality control samples are $9.6 \%, 7.2 \%$ and 5.6\%, respectively. hs-CRP was measured immunochemically in plasma using Siemens reagents on a nephelometer autoanalyzer (BNII). The assay sensitivity is $0.004 \mathrm{mg} / \mathrm{dL}$ and the interassay CVs of the low-, medium-, and high-level quality control samples were consistently $<3 \%$. Fibrinogen was measured immunochemically in plasma using Siemens reagent on a nephelometer autoanalyzer (BNII). The assay sensitivity was $3.0 \mathrm{mg} / \mathrm{dL}$ and the CV consistently $<4 \%$.

\subsection{Potential confounders and effect modifiers}

A Directed Acyclic Graph (DAG) was developed a priori based on the literature and guided our selection of covariates and model tests. Our final DAG (Fig. A1 Appendix) and fully-adjusted models included demographic variables (gender, race/ethnicity, age and socioeconomic position) and lifestyle variables (smoking, physical activity/sedentary behavior). In addition, smoking status, race/ethnicity, statin use and gender were considered as effect modifiers because these variables have been shown to influence results in studies performed in adults (Bravo et al., 2016; Lee et al., 2016; Ostro et al., 2014; Tsai et al., 2012). DAG development also dictated sensitivity analyses in which fasting status was included as an additional confounder in fully-adjusted models and examined as an effect modifier.

Data on all potential confounders and effect modifiers were collected at the baseline study visit via interview-administered questionnaires. For the measure of neighborhood affluence, residential address was used to obtain the percentage below poverty for the census tract of residence using the 2000 Census. For participants aged ten years and older, information on smoking and physical and sedentary activity was assessed with questions based on the CDC-Sponsored Youth Risk Behavior Surveillance System. Questions ascertained whether the participants had any history of smoking tobacco ("even one or two puffs") and among those that did, how many days in the past 30 in which they smoked tobacco. Physical and sedentary activity were assessed via questions about the average time per week spent watching 
television and playing video/computer games, as well as average number of days per week spent exercising or participating in physical activity that made them sweat for at least $20 \mathrm{~min}$.

\subsection{Exposures}

We used spatio-temporal models to provide estimates of 24-hour average $\mathrm{PM}_{2.5}$ mass and weekly average EC concentrations across the conterminous US from 1999 to 2011 (Yanosky et al., 2018). In addition, we used Atmospheric Dispersion Modeling System (ADMS)-Roads software (CERC; Cambridge, England) to provide estimates of trafficrelated PM exposures near roadways. Residential addresses at the time of the baseline study visit were geocoded for each participant using ArcGIS 9.3 software (ESRI 2008) and the TIGER 2000 and 2006 Road Network Files, using a $30 \mathrm{~m}$ offset to account for residential distance from the road. We achieved a street-segment match rate of over $94 \%$. These geocoded addresses were then used to assign exposure estimates to each participant's location for $\mathrm{PM}_{2.5}$ mass, EC, and ADMS-Roads traffic-related PM levels. For ADMS-Roads, traffic-related PM levels were estimated from all roadways including surface streets (US Census Feature Class codes A1-A4 and A6 (hereafter ADMS-Roads A1-A6 exposures)) as well as those from only major roadways (US Census Feature Class codes A1-A3 (hereafter ADMS-Roads A1-A3 exposures)). Moving averages corresponding to the seven days prior to blood draw (referred to as weekly averages) were calculated for each exposure metric. For $\mathrm{PM}_{2.5}$ mass and ADMS-Roads A1-A6, exposures were also calculated for the day of blood draw (lag 0), for each of the 7 days prior to blood draw (lag days 1-7), and for moving averages of 2, 3, 4 and 5 days prior to blood draw.

Details of these models, including full results and a description of cross-validation procedures and reference maps demonstrating spatial resolution, have been published elsewhere (Yanosky et al., 2018). Briefly, $24 \mathrm{~h}$ average $\mathrm{PM}_{2.5}$ concentration data were obtained from the US Environmental Protection Agency (USEPA) Air Quality System (AQS), from the Interagency Monitoring of Protected Visual Environments (IMPROVE) network, and from the Southern Aerosol Research and Characterization Study (SEARCH) network. EC measurements were from the AQS (parameter code 88380) and IMPROVE (parameter code 88321 ) networks for one of every three days and were used to estimate a weekly average. Predictive accuracy of the $\mathrm{PM}_{2.5} \mathrm{EC}$ model ranged from a cross-validation $\mathrm{R}^{2}$ of 0.6 for a weekly average to 0.8 for an annual average. Data on meteorological factors influencing air pollution dispersion including wind speed and direction, sensible heat flux, planetary boundary layer height, air temperature, total precipitation, and total snowfall were obtained from the (MERRA) project (Goddard Earth Sciences Data and Information Services Center, 2010). Data on traffic counts from Geographic Data Technology, Inc. (Lebanon, NH) Dynamap Traffic Counts v4.2 were spatially joined to ESRI StreetMap Pro 2007 to obtain the US Census Feature Class Code (US Census Bureau 2013) road class: A1 (primary roads, typically interstates, with limited access), A2 (primary major, non-interstate roads), A3 (smaller, secondary roads, usually with more than two lanes), A4-6 (smaller roads, traffic circles and vehicle trails for local traffic); the resulting traffic counts were spatially averaged. Data on county-level population density (in persons $\mathrm{m}^{-2}$ ) from the 2000 US Census (US Census Bureau 2013) were obtained from ESRI Data and Maps 10.1, elevation data from the USGS National Elevation Dataset (USGS 2013). The spatiotemporal generalized additive mixed models (GAMMs) for $\mathrm{PM}_{2.5}$ mass and EC had the following generic form (Yanosky et al., 2014):

$y_{i, t}=\alpha+\alpha_{t}+\sum_{q} d_{q}\left(X_{i, q}\right)+\sum_{p} f_{p}\left(Z_{i, t, p}\right)+g_{t}\left(s_{i}\right)+g\left(s_{i}\right)+b_{i}+e_{i, t} ;$

$b_{i} \sim N\left(0, \sigma^{2} b\right) ; e_{i, t} \sim N\left(0, \sigma^{2}{ }_{e t}\right)$

where $y_{i, t}$ are monitor measurements for $i=1 \ldots I$ sites and $t=1 \ldots T 24$ hr time periods, $s_{i}$ is the projected spatial coordinate pair for the $i$ th location. $X_{i, q}$ are GIS-based time-invariant spatial covariates for $q=1 \ldots Q, Z_{i, t, p}$ are spatio-temporal covariates (including ADMS-Roads traffic-related PM levels) for $p=1 \ldots P$, and $\alpha_{t}$ is an intercept representing the adjusted mean across all sites on a given day. $d_{q}$ are penalized spline smooth functions of $Q$ GIS-based time-invariant spatial covariates, $f_{p}$ are penalized spline smooth functions of $P$ spatio-temporal covariates $g_{t}\left(s_{i}\right)$ accounts for residual spatial variability in the 24hr average values, and $g\left(s_{i}\right)$ accounts for time-invariant spatial variability across the conterminous US. The site-specific random effect $b_{i}$ represents unexplained site-specific variability.

\subsection{Statistical analysis}

Of the 3530 participants available for the study from five sites of the SEARCH cohort, we excluded 149 with missing information on all outcomes; 35 with missing or unknown values for age, gender, site, or percentage below poverty for the census tract of residence; 587 with missing or zero values for ADMS-Roads A1-3 exposures due to living $>2 \mathrm{~km}$ from the nearest class A1-3 road (though these participants are included in sensitivity analyses); 6 with missing values for EC exposures; 21 reporting statin medication use; and 166 with geocode quality other than street segment match or better resulting in a final sample size of 2566 . Among this population, 386 were missing only IL- 6 measures, 108 were missing only hs-CRP, and 100 were missing only fibrinogen. After the inflammatory biomarkers' distributions were examined and influential outliers removed, 2179 participants were included in the IL- 6 analyses, 2458 in hs-CRP analyses, and 2466 in fibrinogen analyses. Correlations among exposures are presented in a supplementary table (A1), and correlations among outcomes were: IL-6 with hs-CRP: 0.11 and fibrinogen: 0.05 and hs-CRP with fibrinogen: 0.05 . Based on examinations of exposure and outcome distributions, all were log-transformed. Separate linear mixed models for each exposure and each outcome, with study site as a random effect, were considered in basic models. Models with covariates added one at a time examined the influence of potential confounders identified through the DAG process. Models which included all demographic variables (age, gender, race/ethnicity, and census tract of residence percent below poverty) were considered, followed by fully-adjusted models which included demographic variables as well as lifestyle factors identified by the DAG (ever tried smoking, number of days smoked in past 30, time spent watching television/playing computer/video games, and days of vigorous exercise in past week). All models were evaluated with and without adjustment for fasting status. Interactions by smoking and fasting status, race/ethnicity, and gender were examined via interaction terms in fully-adjusted models, and by stratified models. Due to the small number of participants reporting statin use $(n=21)$, we did not examine it as an effect modifier but excluded those on statins from the analysis. From these models we estimated the percent difference in each outcome (IL-6, hs-CRP, and fibrinogen) for an IQR change of each exposure (weekly average EC and ADMS Roads A1-3, and weekly average and 0-7 day lags for ADMS Roads 1-6 and $\mathrm{PM}_{2.5}$ mass). We conducted sensitivity analyses that included participants with any pollutant exposure data; the majority of whom were missing ADMS Roads A1-3 exposures. All analyses were conducted using SAS 9.4 (Cary, NC).

\section{Results}

As shown in Table 1, approximately $51 \%$ of the 2566 participants were male, and the majority were White $(75.2 \%)$. Colorado provided the greatest number of participants (29.3\%) and South Carolina the least (7.6\%). More than half reported never smoking (54.4\%). Among those who tried smoking, most (62.0\%) only smoked 1 day in the past month. Approximately 38\% percent of participants reported between 1 and 4 days of vigorous physical activity over the past week, and about $89 \%$ reported fasting prior to blood draw. The mean age of participants was 12.3 years (SD: 4.4 ) with $73 \%$ over age 10 years old, mean days per 
Table 1

Descriptive statistics of covariates, inflammatory biomarkers and air pollution exposures in the search cohort participants included in the primary and sensitivity analyses.

\begin{tabular}{lll}
\hline Variable & $\begin{array}{l}\text { Primary analyses } \\
(N=2566)\end{array}$ & $\begin{array}{l}\text { Sensitivity analyses } \\
(N=3129)\end{array}$ \\
\cline { 2 - 3 } & $\begin{array}{l}\text { No. }(\%) \text { or mean } \\
\text { (SD) }\end{array}$ & No. (\%) or mean (SD) \\
\hline
\end{tabular}

\section{Gender}

Female

1245(48.5)

$1518(48.5)$

Race

Black

Hispanic

Other races

White

Site

South Carolina

Ohio

Colorado

California

Washington

Smoking status

Missing

Not asked (under 10)

Never smoker

Tried smoking

Among smokers, days in past 30

2 to 5

6 to 7

Missing

Days in past 7 vigorous exercise

0

$1-4$

5-7

Missing

Not asked (under 10)

Blood draw was fasting

No

Age

234(9.1)

$329(12.8)$

74(2.9)

1929(75.2)

196(7.6)

$543(21.2)$

753(29.3)

$410(16.0)$

664(25.9)

28 (1.1)

715(27.9)

1397(54.4)

426(16.6)

264 (62.0)

78(18.3)

81(19.0)

3(0.7)

235(9.1)

988(38.5)

600(23.4)

28(1.1))

715(27.0)

283(11.0)

12.3(4.4)

$4.0(1.5)$

2.7(1.5)

Days in past 7 computer use (over 9 years)

Percent below poverty in tract of residence

Pollutant exposures avg week prior to blood draw

ADMS roads A1-6 $\mu \mathrm{g} / \mathrm{m}^{3}$

ADMS roads A1-6 (geometric) $\quad 0.09(0.001)$

ADMS roads A1-6 IQR $\mu \mathrm{g} / \mathrm{m}^{3}$

ADMS roads A1-3

ADMS roads A1-3 (geometric)

ADMS roads A1-3 IQR

PM2.5 mass $\mu \mathrm{g} / \mathrm{m}^{3}$

PM2.5 mass (geometric)

PM2.5 mass $\mu \mathrm{g} / \mathrm{m}^{3}$ IQR

EC $n g / \mathrm{m}^{3}$

EC (geometric)

$\mathrm{EC} \mathrm{ng} / \mathrm{m}^{3} \mathrm{IQR}$

0.1

$0.06(0.1)$

$0.02(0.001)$

0.05

$11.2(5.5)$

2.3(0.01)

11.2

$882.8(538.1)$

$6.6(0.01)$

617.2

$0.1(0.1)$

$0.08(0.001)$

0.1

$89(17.6)$

96(19.0)

3(0.01)

293(9.4)

1207(38.6)

$727(23.2)$

$870(27.8)$

32(1.0)

334(11.0)

12.2(4.4)

$4.0(1.5)$

2.7(1.5)

8.3(7.9)

Outcome variables

\begin{tabular}{lcc}
\hline & $N=2179$ & $N=2642$ \\
IL-6 $\mu \mathrm{g} / \mathrm{L}$ & $18.4(24.9)$ & $18.0(24.0)$ \\
IL-6 (geometric) & $0.9(0.01)$ & $0.9(0.01)$ \\
& $N=2458$ & $N=2991$ \\
hs-CRP mg/dl & $1695.0(4680.9)$ & $1667.5(4610.9)$ \\
hs-CRP (geometric) & $6.0(0.03)$ & $6.0(0.03)$
\end{tabular}

Table 1 (continued)

\begin{tabular}{lcc}
\hline Outcome variables & & \\
\hline & $N=2466$ & $N=3001$ \\
Fibrinogen mg/dl & $352.5(72.1)$ & $352.1(71.1)$ \\
Fibrinogen (geometric) & $5.8(0.004)$ & $5.8(0.004)$ \\
\hline
\end{tabular}

week of television watching was 4 (SD: 1.5), mean days per week of computer use was 2.7 (SD: 1.5), and the mean percentage of residents living in poverty in participants' census tract of residence was 8.6\% (SD: $8.3 \%)$.

For the week prior to the blood draw, the geometric mean for ADMS Roads A1-6 traffic-related PM exposure was $0.09 \mu \mathrm{g} / \mathrm{m}^{3}$ (SD: 0.001 ), for ADMS Roads A1-3 exposures: $0.02 \mu \mathrm{g} / \mathrm{m}^{3}$ (SD: 0.001), $2.3 \mu \mathrm{g} / \mathrm{m}^{3}$ (SD: 0.01 ) for $\mathrm{PM}_{2.5}$ mass exposures, and $6.6 \mathrm{ng} / \mathrm{m}^{3}$ (SD: 0.01) for exposure to EC. The IQR for weekly average ADMS Roads A1-6 was $0.1 \mu \mathrm{g} / \mathrm{m}^{3}, 0.05 \mu \mathrm{g} / \mathrm{m}^{3}$ for ADMS Roads A1-3, $11.2 \mu \mathrm{g} / \mathrm{m}^{3}$ for $\mathrm{PM}_{2.5}$ mass exposures, and 617.2 for $\mathrm{EC} \mathrm{ng} / \mathrm{m}^{3}$. The geometric mean for IL-6 was 0.9 (SD: 0.01), 6.0 for hs-CRP (SD: 0.03), and 5.8 (SD: 0.004) for fibrinogen. Sensitivity analyses adding participants who were missing primarily the ADMS Roads A1-3 pollutant exposure (i.e., lived $>2 \mathrm{~km}$ from a major road or at a distance where levels reduced to zero) resulted in a higher percentage of non-Hispanic whites (77.6\%) and participants from South Carolina (e.g. 10.3\%) and somewhat lower mean EC week exposures (822.9 [SD: 522.7$] \mathrm{ng} / \mathrm{m}^{3}$ vs. 882.8 [SD: 538.1] $\mathrm{ng} / \mathrm{m}^{3}$ ).

\section{1. $I L-6$}

In mixed models adjusting only for site (basic models), increased levels of EC exposures averaged over the week prior to blood draw were positively associated with higher IL-6 levels. Comparing EC weekly averages from the 25th to the 75th percentiles, exposure was associated with an 8.3\% higher level of IL-6 (95\%CI: 2.7\%,14.3\%) (Fig. A.2). Similarly, comparing exposure from the lowest to highest quartile of $\mathrm{PM}_{2.5}$ mass exposures on lag day 3, exposure was associated with $5.0 \%$ higher IL-6 level (95\%CI: $-0.01 \%, 10.3 \%)$ in basic models. A negative association was observed with ADMS Roads 1-3 exposures ( $-4.7 \%$ IQR change; 95\%CI: $-9.0 \%,-0.2 \%$ ), which weakened somewhat after adjustment for demographic variables. We observed no other consistent associations in basic models for any of the pollutants we examined. Further adjustment for smoking, physical activity, sedentary behavior, and fasting status (lifestyle factors) did not appreciably change model results. For example, in fully-adjusted models (Fig. 1), comparing exposure from the 25th to 75th percentile of weekly average EC, exposure was associated with an 8.9\% higher IL-6 level (95\%CI: 3.2\%,14.9\%) (Fig. 1). Likewise, comparing exposure from the 25th to 75th percentile of $\mathrm{PM}_{2.5}$ mass exposures on lag day 3 was associated with a $5.4 \%$ higher IL-6 level (95\%CI: 0.4\%,10.7\%). Interactions were observed by race/ ethnicity with non-Hispanic Black participants showing higher IL-6 levels with decreasing $\mathrm{PM}_{2.5}$ mass exposures on lag days 4 through 6 , while non-Hispanic White participants showed higher levels of IL-6 with increasing $\mathrm{PM}_{2.5}$ mass exposures on lag days 3 and 4. Participants of other races also had positive associations for lag days 4 and 7. Participants who were of Hispanic ethnicity or White race showed higher IL-6 levels when exposed to higher levels of EC, as demonstrated in Fig. A3. With an increase from the lowest to highest quartile, Blacks showed a 10.0\% lower IL-6 level (95\%CI: $-27.5 \%, 11.6 \%)$, while Whites and Hispanics showed an 8.4\% (95\%CI: 2.0\%,15.1\%) and 25.9 (95\%CI: $7.0 \%, 48.2 \%$ ) percent higher IL-6 level, respectively. No other consistent, robust interactions were observed. Results were comparable for sensitivity analyses that included participants who were missing any of the individual pollutant exposures or had an equivalent of zero for ADMS Roads A1-3 pollution, meaning that they lived further than $2 \mathrm{~km}$ from a major road. 


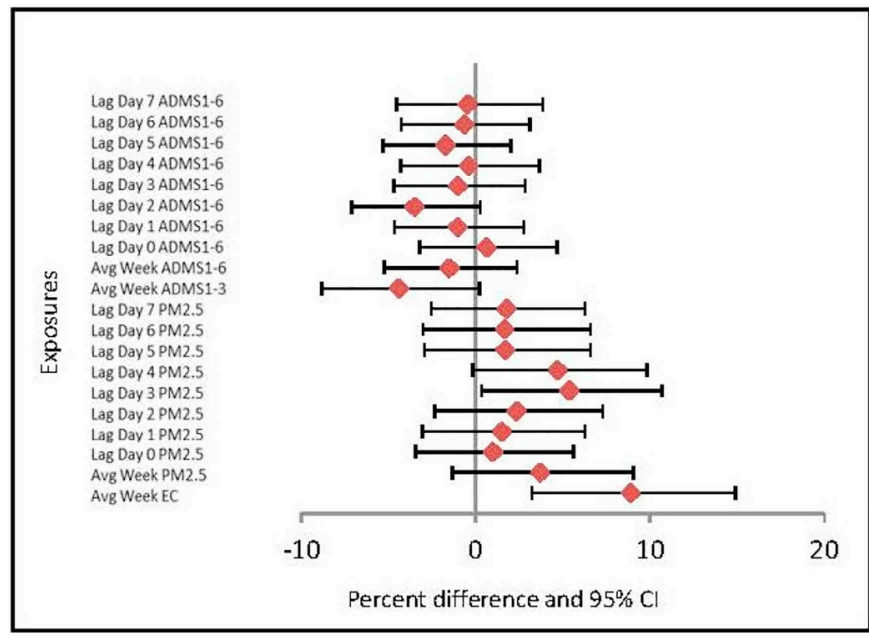

Fig. 1. Percent difference in IL-6 levels with an IQR change in estimated EC, $\mathrm{PM}_{2.5}$ and ADMS roads exposures in fully-adjusted models.

*Model adjusts for site, age, gender, residential census tract percent below poverty, physical activity, sedentary behavior and smoking.

\section{2. $h s-C R P$}

In basic models, we found positive associations for hs-CRP with weekly-average EC exposures (9.8\% higher hs-CRP comparing exposure from the 25th to 75th percentile of EC, 95\%CI: $2.4 \%, 17.7 \%$ ), and with $\mathrm{PM}_{2.5}$ mass exposures on lag days 4 through 6 , as well as the weeklyaverage (Fig. A4). For example, comparing exposure from the 25th to 75th percentile in weekly-average $\mathrm{PM}_{2.5}$ mass exposure was associated with an $8.9 \%$ higher level of hs-CRP (95\%CI: $1.3 \%, 17.0 \%)$. No other consistent associations were observed with other lags for $\mathrm{PM}_{2.5}$ mass or with any lags or the weekly average for ADMS Roads 1-6. Moving average exposures of $\mathrm{PM}_{2.5}$ mass for 2, 3, 4 and 5 days prior to blood draw were also positively associated with higher levels of hs-CRP, however no associations were observed with moving average exposures of ADMS Roads 1-6. Adjusting for demographic variables attenuated the associations for most exposure models, however strong positive associations remained for weekly-average EC. For example, comparing exposure at the 25th to the 75th percentile of EC, exposure was associated with 7.8\% higher hs-CRP level, 95\%CI: $1.2 \%, 14.7 \%$. Additional adjustment for lifestyle factors resulted in comparable findings for all exposure models (Fig. 2). Addition of fasting status to any of the basic, demographic, or fully adjusted models also did not change the results. Examination of interaction tests showed a strong gender difference for $\mathrm{PM}_{2.5}$ mass exposures on lag day 7 , with stratified results revealing weak differences but in the same direction for lag days 4-6 and the weekly average. Higher hs-CRP levels were positively associated with increased $\mathrm{PM}_{2.5}$ mass exposures on lag day 7 among females (comparing exposure in the 25 th to the 75 th percentile, exposure was associated with $8.8 \%$ higher hs-CRP level; 95\%CI: $1.0 \%, 17.2 \%$ ) while a weak inverse relationship was observed among males (comparing exposure at the 25th compared with 75th percentile, exposure was associated with a $6.2 \%$ lower level of hs-CRP; 95\%CI: $-12.9 \%, 1.0 \%$ ) (Fig. A5). Sensitivity analyses (including 587 subjects with zero ADMS-Roads A1-3 exposures) showed the same effect modification by gender for the hs-CRP relationship with $\mathrm{PM}_{2.5}$ mass exposures. In these analyses, the relationship with weekly-average EC was weaker but in the same direction as for the main analysis.

\subsection{Fibrinogen}

Basic models showed a strong positive association between ADMS Roads A1-6 exposure on lag day 1 and higher levels of fibrinogen (Fig. A6), however no associations were observed for any other lags, the

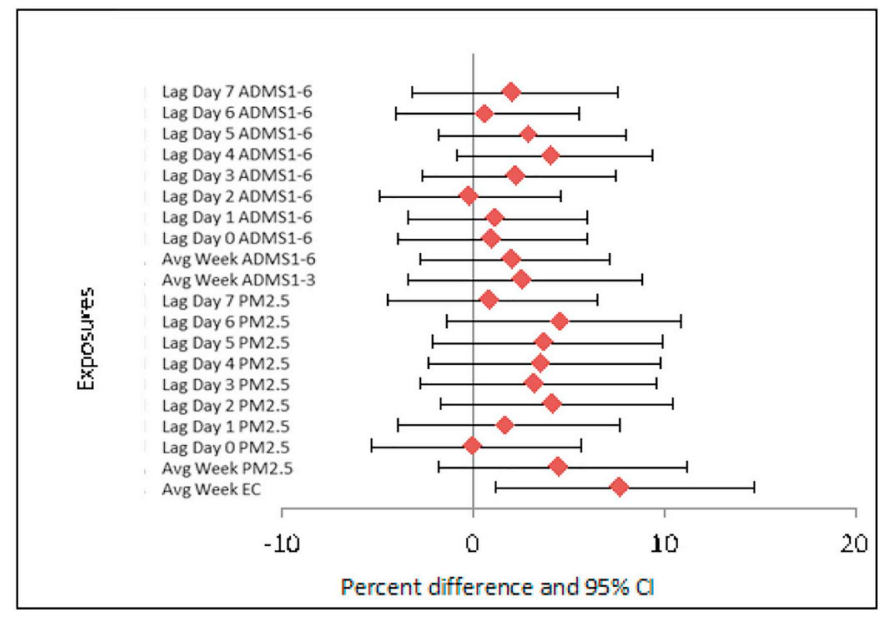

Fig. 2. Percent difference in hs-CRP levels with an IQR change in estimated EC, $\mathrm{PM}_{2.5}$ and ADMS roads exposures in fully-adjusted models.

*Model adjusts for site, age, gender, residential census tract percent below poverty, physical activity, sedentary behavior and smoking.

weekly average or moving averages. Weekly average EC and weekly average ADMS Roads A1-3 were also not associated with fibrinogen levels. We observed strong positive associations between $\mathrm{PM}_{2.5}$ mass exposures for lag days 2 and 3, the weekly-average, and moving averages of 2, 3, 4 and 5 days prior to blood draw with higher levels of fibrinogen. Associations were not observed with other daily lags. Adjustment for demographic and lifestyle variables attenuated these results, with the findings for $\mathrm{PM}_{2.5}$ mass on lag days 2 and 3 remaining weakly positive. For example, as shown in Figs. A6 and 3, comparing the 25th to 75th percentile of exposure to $\mathrm{PM}_{2.5}$ mass on lag day 3, exposure was associated with a $1.0 \%$ higher (95\%CI: $0.2 \%, 1.9 \%)$ level of fibrinogen in basic models, which was attenuated to $0.8 \%$ (95\% CI: $-0.01 \%, 1.57 \%$ ) in fully-adjusted models. Adjustment for fasting status did not change any findings. Interactions were observed by race/ethnicity, but not by smoking, gender or fasting status. Participants reporting Black race showed lower levels of fibrinogen associated with increased ADMS Roads 1-6 exposures on lag days 3 and 6 (Fig. A7). For the same days, White participants showed weak positive relationships. For example, comparing ADMS Roads 1-6 exposures on day 3 in the 25th to 75th percentile, fully-adjusted models showed fibrinogen levels were $2.4 \%$ lower (95\%CI: $-4.4 \%,-0.4 \%$ ) among Blacks, $0.7 \%$ lower

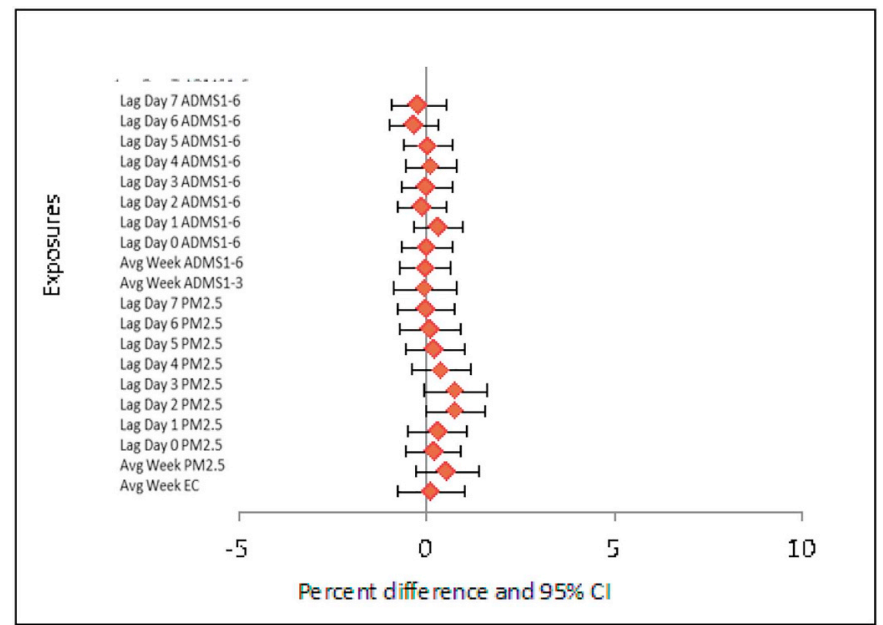

Fig. 3. Percent difference in fibrinogen levels with an IQR change in estimated EC, $\mathrm{PM}_{2.5}$ and ADMS roads exposures in fully-adjusted models *Model adjusts for site, age, gender, residential census tract percent below poverty, physical activity, sedentary behavior and smoking. 
(95\%CI: $-2.4 \%, 1.1 \%$ ) among Hispanics, but were $0.4 \%$ higher (95\%CI: $-0.4 \%, 1.2 \%$ ) among Whites and $2.7 \%$ higher (95\%CI: $-1.1 \%, 6.7 \%)$ among participants of other races. Results from sensitivity analyses were similar.

\section{Discussion}

In a racially and geographically diverse cohort of youth with type 1 diabetes, we observed strong positive associations of EC average week exposures with IL- 6 and hs-CRP in models adjusting for demographic (gender, race/ethnicity, age and neighborhood affluence) and lifestyle variables (smoking, physical activity, sedentary behavior). In addition, higher $\mathrm{PM}_{2.5}$ mass exposures on lag day 3 were positively associated with higher IL-6 levels in fully adjusted models. The relationship between higher $\mathrm{PM}_{2.5}$ mass exposures on lag days 2 and 3 with fibrinogen was suggestive. We observed some evidence of effect modification by race/ethnicity and gender. Among non-Hispanic White and Hispanic participants, we found a positive relationship between IL- 6 and weekly EC exposures. Higher $\mathrm{PM}_{2.5}$ mass exposures were strongly related to lower IL-6 levels among non-Hispanic black participants (lag days 4 through 6), and higher IL-6 levels among non-Hispanic White participants (lag days 3 and 4) and participants of other race, non-Hispanic ethnicity (lag days 4 and 7). Increased $\mathrm{PM}_{2.5}$ mass exposures on lag days 4 through 7 , as well as the weekly-average were associated with higher hs-CRP among females but not among males. Among nonHispanic Black participants, we found an inverse relationship between ADMS Roads 1-6 exposures on lag days 3, 4 and 6 and fibrinogen levels.

Our most robust findings were for EC, a product of primary combustion, with IL- 6 and hs-CRP. IL- 6 is a proinflammatory cytokine and leads to CRP production, which in turn can lead to release of glycoproteins that impact coagulation (Ridker, 2004). Hs-CRP is often used as a marker of clinical risk for atherothrombosis and vascular dysfunction (Ridker, 2016). Very few studies to date have examined the relationship of EC with inflammatory biomarkers, and those have been mostly conducted among healthy adults. In a study of trucking industry workers, Chiu et al. (2016) reported a null association of EC exposures up to 2 days prior to blood draw with IL-6 or hs-CRP (Chiu et al., 2016), whereas we observed a positive association for the weekly-average. Also in contrast to our findings, two studies of daily exposures to EC among healthy adults of working age, reported no associations with hsCRP (Mirowsky et al., 2015; Strak et al., 2013). Exposures for all of these studies were assessed within $48 \mathrm{~h}$ or less of blood draw, whereas EC was averaged over the week prior in the current study. Similar to our findings, Strak et al. (2013) found no association with fibrinogen, a plasma protein activated by IL- 6 and other cytokines and involved in coagulation, thrombosis and possibly the development of atherosclerotic plaque (Ariëns, 2013). Given the paucity of research with EC to date, it is unclear whether EC exerts effects with longer time periods or whether the susceptibility of youth with T1D is greater to EC-related inflammation as related to IL- 6 and hs-CRP markers.

Direct comparisons of our findings with the literature are also limited because the vast majority of research on the relationship of acute air pollutant exposures with inflammatory biomarkers has been conducted among adults without diabetes. However, we identified a study conducted among 374 Iranian youth aged 10-18 without diabetes. The authors reported an association between weekly average $\mathrm{PM}_{10}$ exposure and hs-CRP. $\mathrm{PM}_{2.5}$ was not examined (Kelishadi et al., 2009). We did not find an association between $\mathrm{PM}_{2.5}$ mass exposures and hs-CRP.

The majority of prior research has also focused on PM. We found a strong positive association only between lag day $3 \mathrm{PM}_{2.5}$ mass and IL-6 among youth with type 1 diabetes. Several studies of adults have observed weak or no associations between $\mathrm{PM}_{2.5}$ and IL- 6 with similar time periods. In a repeated measures study of 45 healthy adults, Thompson et al. (2009) found $\mathrm{PM}_{2.5}$ exposures on lag days 0 to 5 was positively, but weakly, associated with IL-6 (Thompson et al., 2009). A study of 115 older women with 3 day average $\mathrm{PM}_{2.5}$ exposures
(Williams et al., 2011) and a study of about 6750 adult Multi-Ethnic Study of Atherosclerosis (MESA) cohort participants using daily averages up to five days prior to blood draw reported no significant associations (Hajat et al., 2015). Similar to our findings, several smaller studies of healthy young adults also found no association of $\mathrm{PM}_{2.5}$ exposures within $24 \mathrm{~h}$ of blood draw and CRP (Bräuner et al., 2008; Mirowsky et al., 2015; Tsai et al., 2012). Two studies of adults with longer exposure averaging times also found no associations, including a study of men in the VA Normative Aging Study (Zeka et al., 2006) with $48 \mathrm{~h}$ or one week average exposures and moving averages up to 5 days in the Heinz Nixdorff recall study of adults (Hertel et al., 2010). We observed weak positive relationships of $\mathrm{PM}_{2.5}$ mass exposures on lag days 2 and 3 with fibrinogen. These findings may be comparable to a study of healthy young adults in Beijing during the Olympics which found a positive but significant association with $\mathrm{PM}_{2.5}$ exposures on lag days two and three (Rich et al., 2012). Also, a panel study of adults with type 2 diabetes and impaired glucose tolerance, Ruckerl and colleagues (2014) found a small positive association between 5 day average $\mathrm{PM}_{2.5}$ exposure and fibrinogen (Rückerl et al., 2014). In contrast, several other acute exposure studies of healthy adults found no links between $\mathrm{PM}_{2.5}$ and fibrinogen (Bräuner et al., 2008; Thompson et al., 2009; Zeka et al., 2006).

Findings differ somewhat for other size fractions of PM. For example, in a study of elderly subjects in the Los Angeles area, Delfino and co-authors (2009) found no association of IL-6 with $\mathrm{PM}_{2.5}$ but significant positive associations with acute $\mathrm{PM}_{0.25}$ exposures (Delfino et al., 2009). No association was observed among adults in the Boston area between particle number concentration (a proxy for ultrafine PM) and IL-6 (Fuller et al., 2015). However, higher acute $\mathrm{PM}_{10}$ exposures were significantly associated with higher IL-6 measures in the CoLaus study of over 6100 adults in Switzerland (Tsai et al., 2012).

Prior studies of cardiovascular-related impacts of $\mathrm{PM}_{2.5}$ mass have shown some differences in gender susceptibility (Bell et al., 2015; Weichenthal et al., 2014). We observed increases of hs-CRP levels with higher exposures to $\mathrm{PM}_{2.5}$ mass exposures at longer lags among female youth with T1D, but similar relationships were not observed among males. However, no effect modification by gender was reported for $\mathrm{PM}_{2.5}$ and CRP for the MESA or Heinz Nixdorff studies among adults (Hajat et al., 2015; Hertel et al., 2010). Further investigation into these potential gender differences is needed.

Though some research has adjusted for confounding by race (generally White versus non-White), most studies have not been able to examine modification by race/ethnicity. We observed some evidence of modification by race/ethnicity for $\mathrm{PM}_{2.5}$ mass and EC with IL- 6 . We found inverse relationships for $\mathrm{PM}_{2.5}$ mass (lags 4-6) among Black participants. However, positive relationships were observed for EC with IL-6 among White participants and Hispanic participants, and with $\mathrm{PM}_{2.5}$ mass among participants of White (lags 3 and 4 ) and other races/ ethnicities (lags 4 and 7). In contrast, a study of about 6750 MESA cohort participants using daily averages up to five days prior to blood draw study found results were comparable among Whites and nonWhites. Chiu et al. (2016) trucking study of mostly male Caucasians did not observe an association between EC and IL-6. Among our unexpected findings, were inverse associations of ADMS Roads A1-6 exposures and fibrinogen among Black and Hispanic youth, while children who were White or of other race/ethnicities showed positive but weak relationships. Most prior research did not present results stratified by race/ ethnicity due to more homogeneous or smaller populations. Whether differences in these findings are due to susceptibility for persons with type 1 diabetes; disparities in age, race/ethnicity, socioeconomic position (SEP) (which are difficult to disentangle in the US); or other factors, should be addressed in further research.

Though the current study takes an important step to address the gap of information regarding the impacts of acute air pollution exposures among youth with type 1 diabetes, the study has limitations. As in much of the previous air pollution research, we were unable to consider 
exposure to second-hand smoke or exposure to noise, which has been linked with diabetes in recent research and which can be correlated with air pollution exposures (Münzel et al., 2017). In addition, we cannot rule out that some of our findings were due to chance. Our hypotheses and the models we identified for testing via development of a DAG were determined a priori. In this initial step to address an understudied topic, we used a qualitative approach to examine and present the overall direction and magnitude of results, and we did not otherwise address multiple comparisons. Thus additional research is needed to confirm our findings, particularly with respect to differences in results by race/ethnicity and gender. Though a strength of this study was the diversity of participant race/ethnicities and SEP levels, residual confounding by SEP and disentangling the contributions of SEP and race/ethnicity presents a challenge for this and other US studies. We addressed potential area-level SEP confounding by including the percent of population living in poverty in each participant's residential census tract. In addition, we conducted a post-hoc analysis adjusting for insurance status and type as a proxy for individual-level SEP and found results were unchanged. We were able to adjust for SEP and a number of important potential confounders identified through our DAG process, however data on physical activity and sedentary behavior were unavailable for participants under age 10. Although, a post-hoc analysis revealed that results did not change with or without this confounder included in the fully-adjusted models. We also had limited information regarding locations of daily activities and thus estimated air pollution exposures only at the residence. However, given the young age of the study population, school and after school activities are likely more proximal to residential addresses than with adult populations. Our air pollution models also enabled us to assess EC, a combustion-related constituent of $\mathrm{PM}_{2.5}$, which few prior studies have been able to examine. Our spatio-temporal statistical exposure modeling process was able to capture the local spatial variation of EC, which has been noted as a potential weakness for other studies that have not observed positive associations between EC and inflammatory biomarkers (MarquesVidal et al., 2013).

\section{Conclusions}

In summary, while prior studies have shown increased air pollutionrelated cardiovascular risks among elderly populations and adults with diabetes (Alexeeff et al., 2011; Devlin et al., 2014; Dubowsky et al., 2006), this is among the first studies to show that a young population, more vulnerable to cardiovascular disease due to T1D, may be at risk for ambient air pollution-related inflammation. Additional research is needed to investigate racial/ethnic and gender differences.

\section{Acknowledgments}

The SEARCH for Diabetes in Youth Study is indebted to the many youth and their families, and their health care providers, whose participation made this study possible. The authors also thank Linda Li, Jun Chu and James Hibbert for their contributions to the SEARCH Air Study.

\section{Grant support}

The SEARCH Air Pollution and Inflammation Ancillary Study is funded by the National Institutes of Health, National Institute of Environmental Health Sciences grant R01 ES019168. SEARCH for Diabetes in Youth is funded by the Centers for Disease Control and Prevention (PA numbers 00097, DP-05-069, and DP-10-001) and supported by the National Institute of Diabetes and Digestive and Kidney Diseases.

\section{Site contract numbers}

Kaiser Permanente Southern California (U18DP006133, U48/ CCU919219, U01 DP000246, and U18DP002714), University of Colorado Denver (U18DP006139, U48/CCU819241-3, U01 DP000247, and U18DP000247-06A1), Children's Hospital Medical Center (Cincinnati) (U18DP006134, U48/CCU519239, U01 DP000248, and 1U18DP002709), University of North Carolina at Chapel Hill (U18DP006138, U48/CCU419249, U01 DP000254, and U18DP002708), Seattle Children's Hospital (U18DP006136, U58/ CCU019235-4, U01 DP000244, and U18DP002710-01), Wake Forest University School of Medicine (U18DP006131, U48/CCU919219, U01 DP000250, and 200-2010-35171).

The authors wish to acknowledge the involvement of the South Carolina Clinical \& Translational Research Institute, at the Medical University of South Carolina, NIH/National Center for Advancing Translational Sciences (NCATS) grant number UL1 TR000062; Seattle Children's Hospital and the University of Washington, NIH/NCATS grant number UL1 TR00423; University of Colorado Pediatric Clinical and Translational Research Center, NIH/NCATS grant Number UL1 TR000154; the Barbara Davis Center at the University of Colorado at Denver (DERC NIH grant number P30 DK57516); the University of Cincinnati, NIH/NCATS grant number UL1 TR000077; and the Children with Medical Handicaps program managed by the Ohio Department of Health. This study includes data provided by the Ohio Department of Health, which should not be considered an endorsement of this study or its conclusions.

The findings and conclusions in this report are those of the authors and do not necessarily represent the official position of the Centers for Disease Control and Prevention or the National Institutes of Health, National Institute of Environmental Health Sciences or National Institute of Diabetes and Digestive and Kidney Diseases.

\section{Appendix A. Supplementary data}

Supplementary data to this article can be found online at https:// doi.org/10.1016/j.envint.2019.105064.

\section{References}

Alexeeff, S.E., Coull, B.A., Gryparis, A., Suh, H., Sparrow, D., Vokonas, P.S., et al., 2011 Medium-term exposure to traffic-related air pollution and markers of inflammation and endothelial function. Environ. Health Perspect. 119, 481-486. https://doi.org/ 10.1289/ehp. 1002560 .

Alman AC, Talton JW, Wadwa RP, Urbina EM, Dolan LM, Daniels SR, et al. 2014. Cardiovascular health in adolescents with type 1 diabetes: The SEARCH CVD Study. Pediatr Diabetes. 15(7): 502-510; doi: 10.1111/pedi.12120.

Ariëns, R.A.S., 2013. Fibrin(ogen) and thrombotic disease. J. Thromb. Haemost. 11 294-305. https://doi.org/10.1111/jth.12229.

Bell, M.L., Son, J.Y., Peng, R.D., Wang, Y., Dominici, F., 2015. Ambient PM2.5 and risk of hospital admissions: do risks differ for men and women? Epidemiology 26 (4), 575-579. https://doi.org/10.1097/EDE.000000000000310.

Bräuner, E.V., M?ller, P., Barregard, L., Dragsted, L.O., Glasius, M., Wahlin, P., et al., 2008. Exposure to ambient concentrations of particulate air pollution does not influence vascular function or inflammatory pathways in young healthy individuals. Part. Fibre Toxicol. 5, 13. https://doi.org/10.1186/1743-8977-5-13.

Bravo, M.A., Anthopolos, R., Bell, M.L., Miranda, M.L., 2016. Racial isolation and exposure to airborne particulate matter and ozone in understudied US populations. Environ. Int. 92-93, 247-255. https://doi.org/10.1016/j.envint.2016.04.008.

Chiu, Y.-H.M., Garshick, E., Hart, J.E., Spiegelman, D., Dockery, D.W., Smith, T.J., et al., 2016. Occupational vehicle-related particulate exposure and inflammatory markers in trucking industry workers. Environ. Res. 148, 310-317. https://doi.org/10.1016/j. envres.2016.04.008.

Delfino, R.J., Staimer, N., Tjoa, T., Gillen, D.L., Polidori, A., Arhami, M., et al., 2009. Air pollution exposures and circulating biomarkers of effect in a susceptible population: clues to potential causal component mixtures and mechanisms. Environ. Health Perspect. 117, 1232-1238. https://doi.org/10.1289/ehp.0800194.

Devlin, R.B., Smith, C.B., Schmitt, M.T., Rappold, A.G., Hinderliter, A., Graff, D., et al., 2014. Controlled exposure of humans with metabolic syndrome to concentrated ultrafine ambient particulate matter causes cardiovascular effects. Toxicol. Sci. Off. J. Soc. Toxicol. 140, 61-72. https://doi.org/10.1093/toxsci/kfu063.

Di Ciaula, A., 2016. Type I diabetes in paediatric age in Apulia (Italy): incidence and associations with outdoor air pollutants. Diabetes Res. Clin. Pract. 111, 36-43. 
https://doi.org/10.1016/j.diabres.2015.10.016.

Dockery, D.W., Pope, C.A., Xu, X., Spengler, J.D., Ware, J.H., Fay, M.E., et al., 1993. An association between air pollution and mortality in six U.S. cities. N. Engl. J. Med. 329, 1753-1759. https://doi.org/10.1056/NEJM199312093292401.

Dubowsky, S.D., Suh, H., Schwartz, J., Coull, B.A., Gold, D.R., 2006. Diabetes, obesity, and hypertension may enhance associations between air pollution and markers of systemic inflammation. Environ. Health Perspect. 114, 992-998.

Fuller, C.H., Williams, P.L., Mittleman, M.A., Patton, A.P., Spengler, J.D., Brugge, D., 2015. Response of biomarkers of inflammation and coagulation to short-term changes in central site, local, and predicted particle number concentrations. Ann. Epidemiol. 25, 505-511. https://doi.org/10.1016/j.annepidem.2015.02.003.

Goddard Earth Sciences Data and Information Services Center, 2010. MDISC Data Subset. Available. https://disc.sci.gsfc.nasa.gov/daac-bin/FTPSubset.pl, Accessed date: 2 July 2017.

Gold, D.R., 2008. Vulnerability to cardiovascular effects of air pollution in people with diabetes. Curr. Diab. Rep. 8, 333-335.

Gurgueira, S.A., Lawrence, J., Coull, B., Murthy, G.G.K., González-Flecha, B., 2002. Rapid increases in the steady-state concentration of reactive oxygen species in the lungs and heart after particulate air pollution inhalation. Environ. Health Perspect. 110, 749-755.

Hajat, A., Allison, M., Diez-Roux, A.V., Jenny, N.S., Jorgensen, N.W., Szpiro, A.A., et al., 2015. Long-term exposure to air pollution and markers of inflammation, coagulation, and endothelial activation: a repeat-measures analysis in the multi-ethnic study of atherosclerosis (MESA). Epidemiology 26, 310-320. https://doi.org/10.1097/EDE. 0000000000000267.

Hamman, R.F., Bell, R.A., Dabelea, D., D’Agostino Jr., R.B., Dolan, L., Imperatore, G., et al., 2014. The SEARCH for diabetes in youth study: rationale, findings and future directions. Diabetes Care 37 (12), 3336-3344.

Hathout, E.H., Beeson, W.L., Nahab, F., Rabadi, A., Thomas, W., Mace, J.W., 2002. Role of exposure to air pollutants in the development of type 1 diabetes before and after $5 \mathrm{yr}$ of age. Pediatr. Diabetes 3, 184-188. https://doi.org/10.1034/j.1399-5448.2002. 30403.x.

Hertel, S., Viehmann, A., Moebus, S., Mann, K., Bröcker-Preuss, M., Möhlenkamp, S., et al., 2010. Influence of short-term exposure to ultrafine and fine particles on systemic inflammation. Eur. J. Epidemiol. 25, 581-592. https://doi.org/10.1007/ s10654-010-9477-x.

Jacobs, L., Emmerechts, J., Mathieu, C., Hoylaerts, M.F., Fierens, F., Hoet, P.H., et al., 2010. Air pollution related prothrombotic changes in persons with diabetes. Environ. Health Perspect. 118, 191-196. https://doi.org/10.1289/ehp.0900942.

Kan, H., London, S.J., Chen, G., Zhang, Y., Song, G., Zhao, N., et al., 2007. Differentiating the effects of fine and coarse particles on daily mortality in Shanghai, China. Environ. Int. 33, 376-384. https://doi.org/10.1016/j.envint.2006.12.001.

Kelishadi, R., Mirghaffari, N., Poursafa, P., Gidding, S.S., 2009. Lifestyle and environmental factors associated with inflammation, oxidative stress and insulin resistance in children. Atherosclerosis 203, 311-319. https://doi.org/10.1016/j.atherosclerosis. 2008.06.022.

Laden, F., Schwartz, J., Speizer, F.E., Dockery, D.W., 2006. Reduction in fine particulate air pollution and mortality: extended follow-up of the Harvard Six Cities study. Am. J. Respir. Crit. Care Med. 173, 667-672. https://doi.org/10.1164/rccm.2005034430C.

Lanzinger, S., Rosenbauer, J., Sugiri, D., Schikowski, T., Treiber, B., Klee, D., Rathmann, W., Holl, R.W., 2018. Impact of long-term air pollution exposure on metabolic control in children and adolescents with type 1 diabetes: results from the DPV registry. Diabetologia 61 (6), 1354-1361. https://doi.org/10.1007/s00125-018-4580-8.

Lee, M., Koutrakis, P., Coull, B., Kloog, I., Schwartz, J., 2016. Acute effect of fine particulate matter on mortality in three Southeastern states from 2007-2011. J Expo Sci Environ Epidemiol 26 (2), 173-179. https://doi.org/10.1038/jes.2015.47.

Malmqvist, E., Larsson, H.E., Jönsson, I., Rignell-Hydbom, A., Ivarsson, S.A., Tinnerberg, H., Stroh, E., Rittner, R., Jakobsson, K., Swietlicki, E., Rylander, L., 2015. Maternal exposure to air pollution and type 1 diabetes-accounting for genetic factors. Environ. Res. 140, 268-274. https://doi.org/10.1016/j.envres.2015.03.024.

Marques-Vidal, P., Bastardot, F., von Känel, R., Paccaud, F., Preisig, M., Waeber, G., et al., 2013. Association between circulating cytokine levels, diabetes and insulin resistance in a population-based sample (CoLaus study). Clin. Endocrinol. 78, 232-241. https:// doi.org $/ 10.1111 / j .1365-2265.2012 .04384$ x.

Maynard, D., Coull, B.A., Gryparis, A., Schwartz, J., 2007. Mortality risk associated with short-term exposure to traffic particles and sulfates. Environ. Health Perspect. 115, 751-755. https://doi.org/10.1289/ehp.9537.

Michalska, M., Bartoszewicz, M., Wąż, P., Kozaczuk, S., Beń-Skowronek, I., Zorena, K. 2017. PM10 concentration and microbiological assessment of air in relation to the number of acute cases of type 1 diabetes mellitus in the Lubelskie Voivodeship. Pediatr Endocrinol Diabetes Metab 23 (2), 70-76. https://doi.org/10.18544/PEDM23.02.0076.

Miller, K.A., Siscovick, D.S., Sheppard, L., Shepherd, K., Sullivan, J.H., Anderson, G.L., et al., 2007. Long-term exposure to air pollution and incidence of cardiovascular events in women. N. Engl. J. Med. 356, 447-458. https://doi.org/10.1056/ NEJMoa054409.

Mirowsky, J.E., Peltier, R.E., Lippmann, M., Thurston, G., Chen, L.-C., Neas, L., et al., 2015. Repeated measures of inflammation, blood pressure, and heart rate variability associated with traffic exposures in healthy adults. Environ. Health 14, 66. https:// doi.org/10.1186/s12940-015-0049-0.

Münzel, T., Sørensen, M., Gori, T., Schmidt, F.P., Rao, X., Brook, J., Chen, L.C., Brook, R.D., Rajagopalan, S., 2017. Environmental stressors and cardio-metabolic disease: part I-epidemiologic evidence supporting a role for noise and air pollution and effects of mitigation strategies. Eur. Heart J. 38 (8), 550-556. https://doi.org/10.1093/ eurheartj/ehw269.

O'Neill, M.S., Veves, A., Zanobetti, A., Sarnat, J.A., Gold, D.R., Economides, P.A., et al., 2005. Diabetes enhances vulnerability to particulate air pollution-associated impairment in vascular reactivity and endothelial function. Circulation 111, 2913-2920. https://doi.org/10.1161/CIRCULATIONAHA.104.517110.

Ostro, B., Broadwin, R., Green, S., Feng, W.-Y., Lipsett, M., 2006. Fine particulate air pollution and mortality in nine California counties: results from CALFINE. Environ. Health Perspect. 114, 29-33.

Ostro, B., Malig, B., Broadwin, R., Basu, R., Gold, E.B., Bromberger, J.T., et al., 2014. Chronic PM2.5 exposure and inflammation: determining sensitive subgroups in midlife women. Environ. Res. 132, 168-175.

Peel, J.L., Metzger, K.B., Klein, M., Flanders, W.D., Mulholland, J.A., Tolbert, P.E., 2007. Ambient air pollution and cardiovascular emergency department visits in potentially sensitive groups. Am. J. Epidemiol. 165, 625-633. https://doi.org/10.1093/aje/ kwk051.

Pereira Filho, M.A., Pereira La, A., Arbex, F.F., Arbex, M., Conceição, G.M., Santos, U.P., et al., 2008. Effect of air pollution on diabetes and cardiovascular diseases in São Paulo, Brazil. Braz. J. Med. Biol. Res. Rev. Bras. Pesqui. Medicas E Biol 41, 526-532.

Pope, C.A., Dockery, D.W., 2006. Health effects of fine particulate air pollution: lines that connect. J. Air Waste Manag. Assoc. 1995 (56), 709-742.

Pope, C.A., Burnett, R.T., Thun, M.J., Calle, E.E., Krewski, D., Ito, K., et al., 2002. Lung cancer, cardiopulmonary mortality, and long-term exposure to fine particulate air pollution. JAMA 287, 1132-1141.

Pope, C.A., Burnett, R.T., Thurston, G.D., Thun, M.J., Calle, E.E., Krewski, D., et al., 2004 Cardiovascular mortality and long-term exposure to particulate air pollution: epidemiological evidence of general pathophysiological pathways of disease. Circulation 109, 71-77. https://doi.org/10.1161/01.CIR.0000108927.80044.7F.

Puett, R.C., Schwartz, J., Hart, J.E., Yanosky, J.D., Speizer, F.E., Suh, H., et al., 2008 Chronic particulate exposure, mortality, and coronary heart disease in the nurses' health study. Am. J. Epidemiol. 168, 1161-1168. https://doi.org/10.1093/aje/ kwn232.

Rich, D.Q., Kipen, H.M., Huang, W., Wang, G., Wang, Y., Zhu, P., et al., 2012. Association between changes in air pollution levels during the Beijing Olympics and biomarkers of inflammation and thrombosis in healthy young adults. JAMA 307. https://doi.org/ 10.1001/jama.2012.3488.

Ridker, P.M., 2004. High-sensitivity C-reactive protein, inflammation, and cardiovascular risk: from concept to clinical practice to clinical benefit. Am. Heart J. 148, S19-S26. https://doi.org/10.1016/j.ahj.2004.04.028.

Ridker, P.M., 2016. From CRP to IL-6 to IL-1: moving upstream to identify novel targets for atheroprotection. Circ. Res. 118, 145-156. https://doi.org/10.1161/ CIRCRESAHA.115.306656.

Rückerl, R., Hampel, R., Breitner, S., Cyrys, J., Kraus, U., Carter, J., et al., 2014. Associations between ambient air pollution and blood markers of inflammation and coagulation/fibrinolysis in susceptible populations. Environ. Int. 70, 32-49. https:// doi.org/10.1016/j.envint.2014.05.013.

SEARCH Study Group, 2004. SEARCH for diabetes in youth: a multicenter study of the prevalence, incidence and classification of diabetes mellitus in youth. Control. Clin. Trials 25, 458-471. https://doi.org/10.1016/j.cct.2004.08.002.

Souza, M.B., Saldiva, P.H., Pope, C.A., Capelozzi, V.L., 1998. Respiratory changes due to long-term exposure to urban levels of air pollution: a histopathologic study in humans. Chest 113, 1312-1318.

Strak, M., Hoek, G., Godri, K.J., Gosens, I., Mudway, I.S., van Oerle, R., et al., 2013. Composition of PM affects acute vascular inflammatory and coagulative markers - the RAPTES project. S.A. Cormiered. PLoS ONE 8, e58944. https://doi.org/10.1371/ journal.pone.0058944.

Tamayo, T., Rathmann, W., Stahl-Pehe, A., Landwehr, S., Sugiri, D., Krämer, U., Hermann, J., Holl, R.W., Rosenbauer, J., 2016. No adverse effect of outdoor air pollution on HbA1c in children and young adults with type 1 diabetes. Int. J. Hyg. Environ. Health 219 (4-5), 349-355. https://doi.org/10.1016/j.ijheh.2016.02.002.

Thompson, A.M.S., Zanobetti, A., Silverman, F., Schwartz, J., Coull, B., Urch, B., et al., 2009. Baseline repeated measures from controlled human exposure studies: associations between ambient air pollution exposure and the systemic inflammatory biomarkers IL-6 and fibrinogen. Environ. Health Perspect. https://doi.org/10.1289/ ehp.0900550.

Tsai, D.-H., Amyai, N., Marques-Vidal, P., Wang, J.-L., Riediker, M., Mooser, V., et al., 2012. Effects of particulate matter on inflammatory markers in the general adult population. Part. Fibre Toxicol. 9, 24. https://doi.org/10.1186/1743-8977-9-24.

Utell, M.J., Frampton, M.W., Zareba, W., Devlin, R.B., Cascio, W.E., 2002. Cardiovascular effects associated with air pollution: potential mechanisms and methods of testing. Inhal. Toxicol. 14, 1231-1247. https://doi.org/10.1080/08958370290084881.

Weichenthal, S., Villeneuve, P.J., Burnett, R.T., van Donkelaar, A., Martin, R.V., Jones, R.R., et al., 2014. Long-term exposure to fine particulate matter: association with nonaccidental and cardiovascular mortality in the agricultural health study cohort. Environ. Health Perspect. 122 (6), 609-615. https://doi.org/10.1289/ehp.1307277.

Williams, L., Ulrich, C.M., Larson, T., Wener, M.H., Wood, B., Chen-Levy, Z., et al., 2011. Fine particulate matter $\left(\mathrm{PM}_{2 \cdot 5}\right)$ air pollution and immune status among women in the Seattle area. Arch. Environ. Occup. Health 66, 155-165. https://doi.org/10.1080/ 19338244.2010.539636.

Yanosky, J.D., Paciorek, C.J., Laden, F., Hart, J.E., Puett, R.C., Liao, D., et al., 2014. Spatio-temporal modeling of particulate air pollution in the conterminous United States using geographic and meteorological predictors. Environ. Health 13, 63. 
https://doi.org/10.1186/1476-069X-13-63.

Yanosky, J.D., Fisher, J., Liao, D., Rim, D., Vander Wal, R., Groves, W., et al., 2018. Application and validation of a line-source dispersion model to estimate small scale traffic-related particulate matter concentrations across the conterminous US. Air Quality, Atmos \& Health 11 (6), 741-754. https://doi.org/10.1007/s11869-0180580-6.

Zanobetti, A., Schwartz, J., 2001. Are diabetics more susceptible to the health effects of airborne particles? Am. J. Respir. Crit. Care Med. 164, 831-833. https://doi.org/10. 1164/ajrccm.164.5.2012039.

Zanobetti, A., Schwartz, J., 2002. Cardiovascular damage by airborne particles: are diabetics more susceptible? Epidemiol. Camb. Mass 13, 588-592. https://doi.org/10. 1097/01.EDE.0000020321.67963.7B.

Zeka, A., Sullivan, J.R., Vokonas, P.S., Sparrow, D., Schwartz, J., 2006. Inflammatory markers and particulate air pollution: characterizing the pathway to disease. Int. J. Epidemiol. 35, 1347-1354. https://doi.org/10.1093/ije/dyl132. 